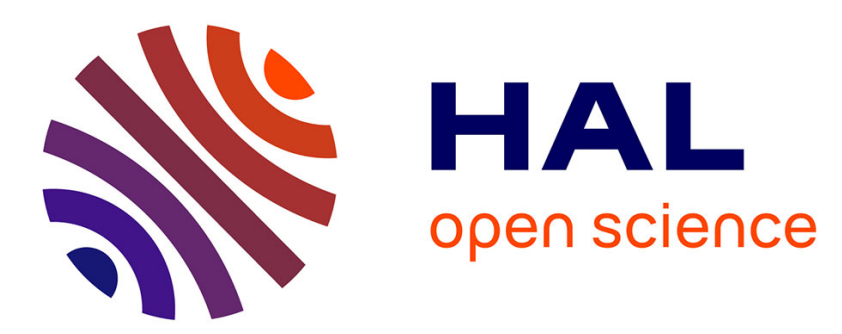

\title{
Controlled Functionalization of Carbon Nanodots for Targeted Intracellular Production of Reactive Oxygen Species
}

Ding-Kun Ji, Giacomo Reina, Shi Guo, Matilde Eredia, Paolo Samorì, Cécilia Ménard-Moyon, Alberto Bianco

\section{To cite this version:}

Ding-Kun Ji, Giacomo Reina, Shi Guo, Matilde Eredia, Paolo Samorì, et al.. Controlled Functionalization of Carbon Nanodots for Targeted Intracellular Production of Reactive Oxygen Species. Nanoscale Horizons, 2020, 5 (8), pp.1240-1249. 10.1039/D0NH00300J . hal-03001556

\section{HAL Id: hal-03001556 https://hal.science/hal-03001556}

Submitted on 16 Nov 2020

HAL is a multi-disciplinary open access archive for the deposit and dissemination of scientific research documents, whether they are published or not. The documents may come from teaching and research institutions in France or abroad, or from public or private research centers.
L'archive ouverte pluridisciplinaire HAL, est destinée au dépôt et à la diffusion de documents scientifiques de niveau recherche, publiés ou non, émanant des établissements d'enseignement et de recherche français ou étrangers, des laboratoires publics ou privés. 


\title{
Controlled Functionalization of Carbon Nanodots for Targeted Intracellular Production of Reactive Oxygen Species
}

\author{
Ding-Kun Ji, ${ }^{1}$ Giacomo Reina, ${ }^{1}$ Shi Guo, ${ }^{1}$ Matilde Eredia,${ }^{2}$ Paolo Samorì, ${ }^{2}$ Cécilia \\ Ménard-Moyon, ${ }^{1}$ Alberto Bianco ${ }^{1, *}$
}
${ }^{1}$ CNRS, Immunology, Immunopathology and Therapeutic Chemistry, UPR 3572, University of Strasbourg, ISIS, 67000 Strasbourg, France
${ }^{2}$ Université de Strasbourg, CNRS, ISIS, 67000 Strasbourg, France




\begin{abstract}
Controlled intracellular release of exogenous reactive oxygen species (ROS) is an innovative and efficient strategy for cancer treatment. Well-designed materials, which can produce ROS in targeted cells, minimizing side effects, still need to be explored as new generation nanomedicines. Here, red-emissive carbon nanodots (CNDs) with intrinsic theranostic properties are devised, and further modified with folic acid (FA) ligand through a controlled covalent functionalization for targeted cell imaging and intracellular production of ROS. We demonstrated that the covalent functionalization is an effective strategy to prevent the aggregation of the dots, leading to superior colloidal stability, enhanced luminescence and ROS generation. Indeed, the functional nanodots own a deep-red emission and good dispersibility in physiological conditions. Importantly, they show excellent targeting properties and generation of high levels of ROS under a $660 \mathrm{~nm}$ laser irradiation, leading to an efficient cell death. These unique properties enable FA-modified carbon nanodots to act as a multifunctional nanoplatform for the simultaneous targeted imaging and efficient photodynamic therapy to induce cancer cell death.
\end{abstract}




\section{Introduction}

Reactive oxygen species (ROS) are well-established key signaling species of cellular stress, damage and death. ${ }^{[1]}$ They include hydrogen peroxide, singlet oxygen, superoxide anion radical, hydroxyl, peroxyl, alkoxyl and hydroperoxyl radicals. ${ }^{[2,3]}$ Since ROS cause oxidative damage of the fatty acids in lipids, the nucleobases in DNA and the amino acids of proteins, leading to cell death by necrosis and/or apoptosis, the controlled intracellular release of exogenous ROS has been employed as an effective strategy for cancer treatment. ${ }^{[4]}$

Light-induced ROS generation can trigger necrosis or apoptosis of cancer cells by eliciting an adequate level of oxidative stress. ${ }^{[5,6]}$ Despite the success of several well-designed materials to achieve this goal, ${ }^{[7,8]}$ the production of ROS in targeted cells, minimizing their damaging effect, has been elusive.

Folate receptor (FR), also known as the high-affinity membrane folate-binding protein, is a glycosylphosphatidylinositol-linked membrane glycoprotein. Two membrane-bound isoforms of FR have been identified as $\alpha$ and $\beta$ with high affinity to folic acid (FA) $\left(K_{D} \sim 0.1 \mathrm{nM}\right) .{ }^{[9,10]}$ FRs are highly expressed in various types of human cancers, and generally absent in most normal cells. ${ }^{[11,12]}$ Therefore, FRs are ideal biomarkers for targeted cancer diagnosis and therapy. Taking into account the affinity between FA and FRs, it has been well confirmed that nanoparticles functionalized with FA can improve endocytosis of cancer cells. ${ }^{[13]}$ In this context, FA-modified nanoparticles have been thoroughly explored for intracellular payload delivery leading to a minimum drug dose administration, less side effects and an improved therapeutic efficacy. ${ }^{[14,15]}$ Consequently, FA-modified nanoparticles show a great application potential for targeted disease theragnosis. 
Carbon nanodots, as rising stars in the carbon nanomaterial family, have drawn tremendous attention in the last years, ${ }^{[16]}$ by virtue of their numerous merits including a tunable emission, a high photostability, a low toxicity, a superior biocompatibility and a rather inexpensive cost in large scale production. ${ }^{[17-19]}$ Thanks to the presence of abundant reactive groups on their surface, the properties of CNDs can be well regulated through chemical functionalization. ${ }^{[20,21]}$ CNDs have demonstrated to be a promising candidate in various biomedical applications. ${ }^{\text {[22-24] }}$ In particular, near-infrared (NIR)-emissive or red-emissive CNDs (RCNDs), which can be excited by long-wavelength lights, show great potential in the biomedical field because of their good tissue penetration. ${ }^{[25,26]}$ Recent literature witnesses an extension of RCNDs to the field of phototherapy because of their unique photophysical properties and biocompatibility. ${ }^{[27-30]}$ However, it is still highly desired to develop synthetic methods to prepare and functionalize RCNDs as their potential for targeted intracellular production of ROS and possible use in photodynamic therapy (PDT) has been rarely explored.

PDT is a medical modality that is currently widely used in clinics. ${ }^{[31]}$ However, the current FDA approved photosensitizers lack of specificity (low targeting) and requires high light dose irradiation to be effective. In this context, new photosensitizers able to target tumor cells and requiring low energy irradiation are highly desirable.

Here, we explored a method to prepare RCNDs and we covalently functionalized RCNDs with FA ligand for the targeted generation of ROS in a cell line that highly expresses FA receptors (Figure 1). A certain number of techniques were used to monitor the preparation process, validating our controllable strategy to prepare multifunctional RCND nanoparticles with improved properties. We demonstrate that the covalent modification of RCNDs with FA 
effectively suppressed the aggregation of the starting carbon nanodots, leading to an enhanced fluorescence emission and increased production of ROS. The as-prepared functional RCNDs display a broad UV absorption from 300 to $800 \mathrm{~nm}$ and a red emission from 500 to $800 \mathrm{~nm}$. Finally, they exhibit a high capacity to target cells via ligand-receptor interaction and they produced ROS upon irradiation at $660 \mathrm{~nm}$, thereby inducing an effective cell death, ultimately confirming their biomedical potential as a versatile, highly efficient photosensitizer for photodynamic cancer therapy.

\section{Results and discussion}

\subsection{Design, synthesis and characterization of RCND-TEG-FA}

The RCNDs were prepared through the hydrothermal treatment of conjugated polythiophene phenylpropionic acid (PPA) as illustrated in Scheme 1. To obtain the precursor PPA molecule, a Suzuki coupling between 3-(4-bromophenyl) propanoic acid and 3-thiophene boronic acid was performed. The carboxyl group in 3-(4-bromophenyl) propanoic acid was first protected by benzyl bromide. The resulting compound 1 further reacted with 3-thiophene boronic acid via a successful Suzuki coupling yielding the protected monomer (2) of PPA. The benzyl group was next hydrolyzed in basic conditions to obtain the desired deprotected compound 3 . RCND precursor PPA was synthesized via oxidative polymerization of $\mathbf{3}$ and then the polymer was hydrothermally carbonized to form water-dispersible RCNDs. The high resolution transmission electron microscopy (HRTEM) images shows that RCNDs own a size of below $5 \mathrm{~nm}$ with a well-resolved crystal lattice (Figure 2a). Selected area diffraction (SAED) pattern further confirmed the high crystalline nature of the RCNDs. In addition, the 
presence of oxygen-containing groups $\left(\mathrm{OH}, \mathrm{COO}^{-}\right)$were demonstrated by FT-IR analysis

(Figure 2c). The percentages of $\mathrm{C}, \mathrm{H}, \mathrm{S}$ and $\mathrm{O}$ were measured via element analysis and corresponded to $56.1 \%, 3.9 \%, 12.4 \%, 27.6 \%$, respectively. All results indicated that S-doped RCNDs with $\mathrm{COOH}$ groups on their surface were well prepared.

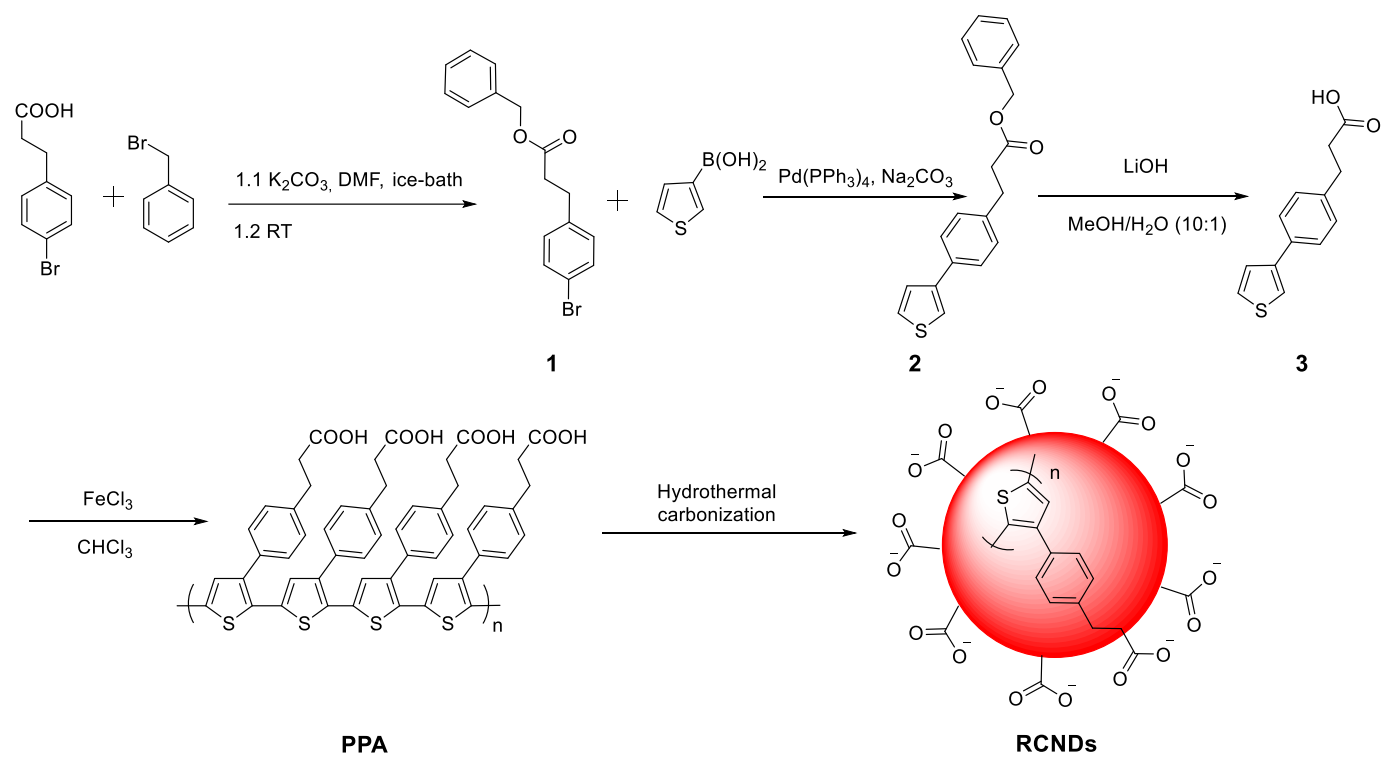

Scheme 1. Synthetic pathway to prepare RCNDs.

It is widely recognized that the hydrothermal technology is a powerful protocol to prepare nanomaterials. ${ }^{[32,33]}$ The hydrothermal treatment is an easy way to transform different conjugated polymers into small-size carbon nanodots endowed of different types of functional groups. The abundant oxygenated groups present on their surface allow a wide range of reactions for the post-modification of CNDs. However, the use of conjugated polymers as precursors to prepare RCNDs with specific functional groups is still not completely understood and fully explored. Here, we attempted to explore tailored RCNDs for biomedical applications.

After obtaining the starting RCNDs, we designed and synthesized the FA ligand according to 
the path outlined in Scheme 2. A short amine-terminated triethylene glycol (TEG) was chosen as a linker to connect FA and RCNDs. Its hydrophilic character can enhance the FA water-dispersibility upon functionalization. The use of short ethylene glycol chains offers also the advantage of minimizing adsorption phenomena and facilitating the removal of unreacted precursors, compared to longer ethylene glycol chains. ${ }^{[34]}$ Compound $\mathbf{4}$ was synthesized by Boc-monoprotection of commercially available $2,2^{\prime}$ '(ethylenedioxy)bis(ethylamine). ${ }^{[35]}$ Folic acid was conjugated to compound $\mathbf{4}$ by amidation through $N$-hydroxysuccinimide (NHS)/1-ethyl-3-(3-dimethylaminopropyl)carbodiimide (EDC) activation. After Boc cleavage using trifluoracetic acid, FA ligand $\mathbf{6}$ was obtained in $27 \%$ total yield. Though FA has two free $\mathrm{COOH}$ groups in the glutamic acid moiety, it has already been reported that the $\gamma-\mathrm{COOH}$ is more prone to amidation than the $\alpha-\mathrm{COOH}$ due to its higher reactivity. ${ }^{[36,37]}$

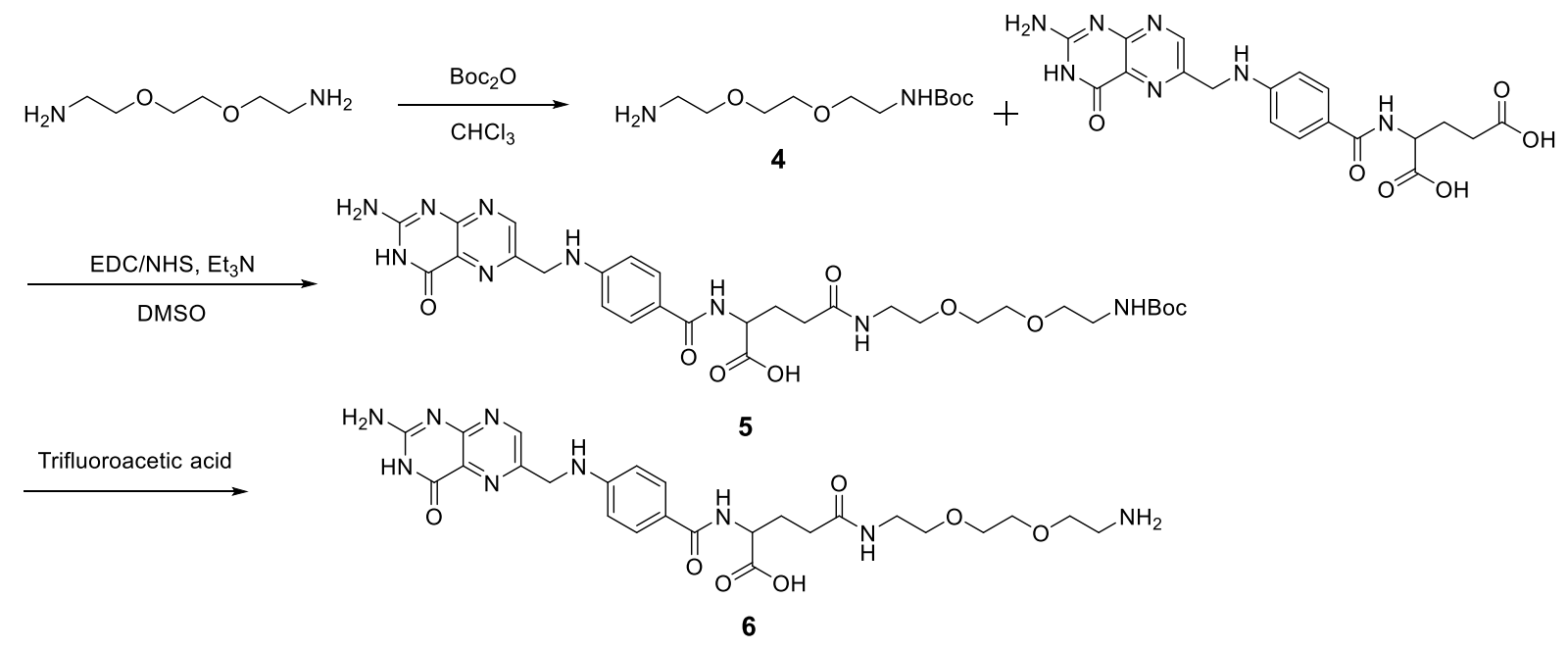

Scheme 2. Synthetic pathway to prepare FA ligand. For simplicity we have represented only the structure of the ligand with the aminated $\gamma-\mathrm{COOH}$.

RCND-TEG-FA was prepared via amidation of the carboxyl groups of RCNDs with the primary amine of FA derivative in the presence of EDC. The formation of RCND-TEG-FA 
was confirmed by FT-IR, UV-Vis, and X-ray photoelectron spectroscopy (XPS). The XPS survey spectra evidenced the presence of carbon, sulfur, oxygen, and nitrogen in the RCND-TEG-FA (Figure 2e). Compared to the XPS of RCNDs (Figure S1), the additional peak of nitrogen confirmed the conjugation with FA ligand. The deconvolution of the $\mathrm{C} 1 \mathrm{~s}$ peak displays five different peaks of carbon atoms, namely the graphitic $\mathrm{C}=\mathrm{C}$, the aliphatic $\mathrm{C}-\mathrm{C}$, the various oxygenated $\mathrm{C}-\mathrm{O}, \mathrm{C}=\mathrm{O}$, and $\mathrm{COO}$, the sulfur $\mathrm{C}-\mathrm{S}$ and the amide $(\mathrm{NH}-\mathrm{C}=\mathrm{O})$ bonds (Figure 2f). FT-IR confirmed the presence of N-H and the amide I stretching at 3296 $\mathrm{cm}^{-1}$ and $1640 \mathrm{~cm}^{-1}$, respectively (Figure 2c). Thus, these results collectively confirm that FA ligand was successfully covalently linked onto the surface of the RCNDs. Thermogravimetric analysis (TGA) of RCND-TEG-FA performed in $\mathrm{N}_{2}$ showed an increase of $10 \%$ weight loss at $600^{\circ} \mathrm{C}$ in comparison to the RCNDs, also confirming the efficiency of FA functionalization (Figure 2d). Finally, the size of as-prepared RCND-TEG-FA was measured by HRTEM and atomic force microscopy (AFM) (Figure 2b). The obtained nanoparticles display a nearly round shape with a lateral size of $\sim 5 \mathrm{~nm}$ and a height of 1 to $5 \mathrm{~nm}$, respectively. Such a narrow size distribution is consistent with graphene quantum dots, ${ }^{[38]}$ and PEG-modified carbon nanodots. ${ }^{[39]}$

\subsection{Colloidal stability of RCNDs and RCND-TEG-FA}

A high colloidal stability in an aqueous medium is essential for evaluating the biological applications of nanoparticles and nanomaterials. ${ }^{[40]}$ In fact, their aggregation can jeopardize many desirable therapeutic performances, including high drug loading capacity and efficient

cellular uptake. ${ }^{[41,42]}$ Even worse, the aggregated nanoparticles can cause hemolysis and tissue 
damage. ${ }^{[43-45]}$ To verify the colloidal stability, $100 \mu \mathrm{g}$ of RCNDs and RCND-TEG-FA were dispersed in $1 \mathrm{~mL}$ of deionized (DI) water, phosphate-buffered saline (PBS) without $\mathrm{Ca}^{2+}$ and $\mathrm{Mg}^{2+}$, saline solution (0.9\%), and cell culture medium (Figure S2). A high colloidal stability of RCND-TEG-FA in both simple and complex physiological media was found (Figure S2a). No precipitates were observed after storing the solutions for 4 days. The concentration of RCND-TEG-FA can reach $1 \mathrm{mg} \cdot \mathrm{mL}^{-1}$ in cell culture medium without any aggregation (Figure S2c), while pristine RCNDs exhibited a bad colloidal stability in the different media (Figure S2b). We further confirmed the aggregation and the increase of the size of the RCNDs using HRTEM, UV-Vis spectra and dynamic light scattering (DLS). From the HRTEM images (Figure S3), we observed a strong aggregation of RCNDs in water, while no aggregation was observed in RCND-TEG-FA sample. We also observed a red shift in UV-Vis absorbance of redispersed RCNDs (Figure 3a), which is a sign of aggregation. DLS showed that monodisperse RCNDs owned a good dispersibility with a narrow size distribution of 4-10 nm (Figure 3b). However, after lyophilization the redispersed RCNDs revealed a strong aggregation with an abnormal size range from 400 to $700 \mathrm{~nm}$ by DLS (Figure 3b). The RCND-TEG-FA displays an increased size than monodisperse RCNDs. All the results indicated that pristine RCNDs without modification are easy to aggregate in neutral solution. The large number of hydroxyl, carbonyl, and carboxyl groups on the surface of RCNDs strongly interacts via hydrogen bonding, thus reducing the repelling forces between the dots, and makes the nanoparticles to cluster into large aggregates. ${ }^{[46]}$ In addition, the lyophilization process likely enhances the aggregation through $\pi-\pi$ stacking between RCNDs. Since it is widely accepted that the size and morphology of nanomaterials play crucial roles in the 
biomedical field, ${ }^{[47-49]}$ there is a real risk that the aggregation may affect their performance similarly to other carbon materials. Fortunately, the aggregated architecture can be prevented by a covalent modification of RCNDs. After amidation with FA ligand, the nanoparticles resulted well-dispersed in water, even after lyophilization, with a slight increased size to 15-30 nm and similar UV-Vis spectra as monodispersed RCNDs. Indeed, the covalent functionalization of RCNDs with FA ligand greatly inhibits their aggregation in physiological environment, allowing to obtain stable solutions that have potential biomedical applications.

\subsection{Fluorescence detection and ROS generation}

With RCND-TEG-FA in hand, we decided to investigate their fluorescence emission for cell imaging and their ROS production. Figure 3d displays the fluorescence spectra of RCNDs and RCND-TEG-FA aqueous solutions. Both samples exhibited a wide range emission spectrum from 500 to $800 \mathrm{~nm}$ with a strong peak at $640 \mathrm{~nm}$ (excitation: $485 \mathrm{~nm}$ ). However, RCND-TEG-FA showed a remarkably stronger fluorescence emission, being $\sim 6$-fold higher than that of RCNDs in water (at $25 \mu \mathrm{g} \cdot \mathrm{mL}^{-1}$ ). Furthermore, the fluorescence quantum yield of RCNDs was measured using a spectrometer attached to an integrating sphere and corresponded to $0.4 \%$ in water, while the fluorescence quantum yield of RCND-TEG-FA increased to $1 \%$. This quantum yield is higher than Se-doped CDs $(0.2 \%)$ and CD nanoparticles $(0.15 \%)$ prepared from polythiophene derivatives, ${ }^{[50,51]}$ Although recently reported F-doped carbon dots were endowed of a high fluorescence quantum yield, ${ }^{[52]}$ the low quantum yield of our RCNDs does not prevent their use for biomedical applications, including fluorescence imaging. ${ }^{51,52]}$ This enhancement of RCND fluorescence is clearly due 
to the presence of the FA ligand preventing RCND aggregation and improving the solubility. These results clearly indicate that the chemical functionalization of the RCNDs improved their fluorescence properties. The enhanced fluorescence intensity could make RCND-TEG-FA useful for cell imaging.

Then, we focused our attention on the generation of ROS by RCND-TEG-FA. ROS were measured by using dihydrorhodamine 123 (DHR123) as the indicator (Figure S4). This non-fluorescent dye becomes rhodamine 123 with a high red emission upon reaction with ROS. The aqueous solution containing RCND-TEG-FA $\left(25 \mu \mathrm{g} \cdot \mathrm{mL}^{-1}\right)$ showed an increase of the fluorescence intensity at $530 \mathrm{~nm}$ upon exposure to a $660 \mathrm{~nm}$ low power laser $\left(0.1 \mathrm{~W} \cdot \mathrm{cm}^{-2}\right)$ at different irradiation times, which was not the case with RCNDs (Figure 3e). The remarkable fluorescence intensity was $\sim 40$-fold higher than that generated by the same concentration of pristine RCNDs. As control, the exposure of an aqueous solution of DHR123 to $660 \mathrm{~nm}$ laser did not induce obvious fluorescence changes, indicating a fast and efficient ROS generation by RCND-TEG-FA. This notable increase of the ROS generation can be ascribed to the higher quantum yield of the functionalized material compared to pristine RCNDs. Altogether, these results reveal that controlled functionalization of FA ligands is an effective strategy to prevent the aggregation of nanodots, leading to a superior colloidal stability, enhanced luminescence and ROS generation (Figure 3e).

To investigate the mechanism of ROS generation, we used thiourea and glutathione (GSH) as inhibitors of $\mathrm{OH}$ and $\mathrm{O}_{2}{ }^{--}$, respectively. ${ }^{[53-55]}$ As shown in Figure $\mathrm{S} 5$ neither thiourea nor GSH were able to inhibit DHR123 oxidation. These results suggest that the hydroxyl radical and the superoxide were not generated during the photo-irradiation of RCND-TEG-FA. Moreover, 
in de-aerated solution, RCND-TEG-FA are also not able to generate ROS indicating that DHR123 oxidation is mediated by $\mathrm{O}_{2}$ radical formation. Radical oxygen formation $\left({ }^{1} \mathrm{O}_{2}\right)$ was evaluated using the specific probe 9,10-anthracenediyl-bis dimalonic acid (ABDA) (Figure 4b). RCND-TEG-FA were able to efficiently oxidize ABDA after laser irradiation. The singlet oxygen quantum yield was further calculated in water by using methylene blue as the standard photosensitizer $\left({ }^{1} \mathrm{O}_{2}\right.$ quantum yield $\Phi_{\mathrm{MB}}=0.52$ in water $)$ and corresponded to 0.40 (Figure S7). ${ }^{[56,57]}$ The yield is similar to the recently reported CD nanopheres $(0.45) .{ }^{[58]}$ Inhibition test irradiating RCND-TEG-FA in the presence of $\mathrm{NaN}_{3}$ as singlet oxygen inhibitor was also performed (Figure 4c). Negligible ABDA oxidation was recorded in the presence of the inhibitor. ABDA oxidation mechanism is normally triggered by singlet oxygen (via an energy transfer mechanism) or superoxide (via a charge transfer mechanism). ${ }^{[59]} \mathrm{As} \mathrm{NaN}_{3}$ quenches only singlet oxygen, the results suggest that only ${ }^{1} \mathrm{O}_{2}$ is formed during the irradiation, while superoxide is again absent, confirming the data described above using GSH inhibitor. Singlet oxygen is commonly produced via type 2 photosensitizers through an energy transfer mechanism. In our case, we used a radical trap (ABDA) well-known to be specific for singlet oxygen detection. The mechanism can be ascribed to the energy transfer as described by other type 2 photosensitizers. From these results we can conclude that ${ }^{1} \mathrm{O}_{2}$ is generated via the typical energy transfer mechanism between the triplet state of RCND-TEG-FA and ${ }^{3} \mathrm{O}_{2}$ that is excited to its singlet state. ${ }^{[2]}$

\subsection{Cellular uptake and fluorescence cell imaging}

To test the receptor-targeted imaging capacity of RCND-TEG-FA, HeLa cells were chosen as 
a model because of their high expression level of FRs. HeLa cells were incubated with RCND-TEG-FA at the concentration of $20 \mu \mathrm{g} \cdot \mathrm{mL}^{-1}$ or $100 \mu \mathrm{g} \cdot \mathrm{mL}^{-1}$ at different time points $(0$, $4,8,24 \mathrm{~h})$. Because of their red emission, RCND-TEG-FA were easily visualized by confocal microscopy. CellMask green was used to observe the morphology of the cell membranes. As shown in Figure 5, RCND-TEG-FA were able to be internalized in HeLa cells already after 4 $\mathrm{h}$, displaying a remarkable red emission. After $8 \mathrm{~h}$ incubation, the cytoplasm of the cells was full of red dots. RCND-TEG-FA displayed a stable fluorescent signal, which was clearly observed into the cells even after $24 \mathrm{~h}$ incubation. To prove the FR-mediated penetration, a competition assay showed that the pre-treatment of HeLa cells with FA resulted in a minimized uptake of RCND-TEG-FA, confirming a FR-mediated endocytosis (Figure 6). These results are consistent with previous reports. ${ }^{[60]}$

We also found that the pristine RCNDs strongly aggregated outside the cells even at a low concentration $\left(20 \mu \mathrm{g} \cdot \mathrm{mL}^{-1}\right)$, which was not the case for RCND-TEG-FA in similar conditions. This is in agreement with the limited colloidal stability of the RCNDs, demonstrating again the importance of the covalent modification of RCNDs.

\subsection{Intracellular detection of ROS}

Inspired by the above results, we attempted to assess the ROS producing ability of RCND-TEG-FA in the targeted cells. To capture the ROS in HeLa cells, CellROX reagent was chosen as indicator. This dye is initially non-fluorescent in a reduced state and becomes fluorescent (yellow color) upon oxidation by ROS. ${ }^{[61]}$ The HeLa cells were incubated with RCND-TEG-FA (at $100 \mu \mathrm{g} \cdot \mathrm{mL}^{-1}$ ) for $24 \mathrm{~h}$ at $37^{\circ} \mathrm{C}$. After washing to remove RCND-TEG-FA 
outside the cells, CellROX reagent was added to monitor the intracellular generation of ROS. We observed that the ROS level was negligible until the laser irradiation was switched on (Figure 7a, 7b). HeLa cells showed strong fluorescence after 10 min irradiation using a low power laser $\left(0.1 \mathrm{~W} \cdot \mathrm{cm}^{-2}\right)$ at $660 \mathrm{~nm}$. As control, HeLa cells without RCND-TEG-FA incubation were also exposed to the same laser irradiation, and no fluorescence changes were observed (Figure S8). These results provide evidence that RCND-TEG-FA are able to produce high level of ROS under irradiation in the visible region, resulting in potential promising tools for PDT. ${ }^{[62]}$

\subsection{In vitro red-light responsive PDT}

To explore the potential of functionalized RCNDs for in vitro red-light responsive PDT, the cytotoxicity and PDT efficacy of RCND-TEG-FA were quantitatively analyzed by alamarBlue ${ }^{\mathrm{TM}}$ cell viability assay. RCND-TEG-FA tested at different concentrations (0-100 $\mu \mathrm{g} \cdot \mathrm{mL}^{-1}$ ) without laser irradiation exhibited negligible toxicity to HeLa cells (Figure $7 \mathrm{c}$ ). This result indicated that RCND-TEG-FA cannot induce cancer cell death, confirming a good biocompatibility. However, the viability of the cells incubated with RCND-TEG-FA (at 0, 20, 50 and $\left.100 \mu \mathrm{g} \cdot \mathrm{mL}^{-1}\right)$ under laser irradiation at $660 \mathrm{~nm}\left(0.1 \mathrm{~W} \cdot \mathrm{cm}^{-2}\right)$ gradually decreased as RCND-TEG-FA concentration increased (Figure 7d). A statistically significant mortality rate of $\sim 70 \%$ was observed at $100 \mu \mathrm{g} \cdot \mathrm{mL}^{-1}$ of RCND-TEG-FA, indicating that RCND-TEG-FA produce a sufficient amount of ROS to kill the cancer cells. The cell viability assay clearly demonstrated that RCND-TEG-FA can be applied as a versatile, highly efficient photosensitizer for simultaneous in vitro red-light responsive fluorescence imaging and PDT. 
A few studies have reported the synthesis of carbon nanodots with intrinsic properties for ROS generation and used as PDT agent. ${ }^{[22,63]}$ However, the targeted release of toxic levels of ROS produced by red-emissive carbon nanodots without loading additional photosensitizers, leading to cell death, has not been reported so far. FA-modified RCNDs showed great capacity to target FR overexpressing cells. Our strategy is flexible as a wide variety of other multifunctional carbon nanodots could be developed for targeted disease imaging and therapy by appropriate covalent functionalization. Other targeting agents such as glycoligands, peptides, aptamers, and small molecules, might expand the use of carbon nanodots.

\section{CONCLUSION}

In summary, we have developed a new method to synthesize red-emissive carbon nanodots using conjugated polythiophenes as precursors, and further modified the nanomaterial with FA for targeted cell imaging and intracellular production of ROS. Many techniques were used to monitor the preparation process, validating our controlled strategy to prepare multifunctional carbon nanodots. We discovered that the covalent functionalization of RCNDs can effectively prevent the aggregation of the dots, leading to superior colloidal stability, enhanced luminescence and ROS generation. Conjugation with folic acid has resulted in a material with very good cancer cell targeting capabilities. The collective properties of FA-modified RCNDs make them a multifunctional nanoplatform for the simultaneous targeted imaging and highly efficient PDT agent for cancer treatment. Our approach might open new ways to the development of a large variety of highly dispersible 
multifunctional carbon nanodots for phototherapies. The synthesis of nanomaterials with intrinsic therapeutic properties offers opportunities for combined therapies through the conjugation of anticancer drugs, nucleic acids, or photothermal agents. 


\section{Conflicts of interests}

The authors declare no conflicts of interest.

\section{Acknowledgments}

We gratefully acknowledge the Centre National de la Recherche Scientifique (CNRS), the International Center for Frontier Research in Chemistry (icFRC), and financial support from the Agence Nationale de la Recherche (ANR) through the LabEx project Chemistry of Complex Systems (ANR-10-LABX-0026_CSC) within the Investissement d'Avenir program (ANR-10-120 IDEX-0002-02). We wish to thank A. Aliprandi for the quantum yield measurements, and C. Royer and V. Demais for help with TEM analyses at the "Plateforme Imagerie in vitro" at the Center of Neurochemistry (INCI, Strasbourg, France). 


\section{References}

[1] D. Trachootham, J. Alexandre, P. Huang, Nat. Rev. Drug Discov. 2009, 8, 579.

[2] Z. Zhou, J. Song, L. Nie, X. Chen, Chem. Soc. Rev. 2016, 45, 6597.

[3] X. Chen, F. Wang, J. Y. Hyun, T. Wei, J. Qiang, X. Ren, I. Shin, J. Yoon, Chem. Soc. Rev. 2016, 45, 2976.

[4] a) D.-K. Ji, C. Ménard-Moyon, A. Bianco, Adv. Drug Deliv. Rev. 2019, 138, 211. b) S. S. Lucky, K. C. Soo, Y. Zhang, Chem. Rev. 2015, 115, 1990.

[5] D.-K. Ji, Y. Zhang, Y. Zang, J. Li, G.-R. Chen, X.-P. He, H. Tian, Adv. Mater. 2016, 28, 9356.

[6] D. E. J. G. J. Dolmans, D. Fukumura, R. K. Jain, Nat. Rev. Cancer 2003, 3, 380.

[7] R. Kurapati, K. Kostarelos, M. Prato, A. Bianco, Adv. Mater. 2016, 28, 6052.

[8] (a) L. Cheng, C. Wang, L. Feng, K. Yang, Z. Liu, Chem. Rev. 2014, 114, 10869; (b) S. Gai, G. Yang, P. Yang, F. He, J. Lin, D. Jin, B. Xing, Nano Today 2018, 19, 146.

[9] J. Sudimack, R. J. Lee, Adv. Drug Deliv. Rev. 2000, 41, 147.

[10] C. P. Leamon, J. A. Reddy, Adv. Drug Deliv. Rev. 2004, 56, 1127.

[11] Y. Lu, E. Sega, C. P. Leamon, P. S. Low, Adv. Drug Deliv. Rev. 2004, 56, 1161.

[12] Y. Lu, P. S. Low, Adv. Drug Deliv. Rev. 2012, 64, 342.

[13] L. Huang, Z. Li, Y. Zhao, J. Yang, Y. Yang, A. I. Pendharkar, Y. Zhang, S. Kelmar,

L. Chen, W. Wu, J. Zhao, G. Han, Adv. Mater. 2017, 29, 1604789.

[14] E. Nogueira, A. C. Gomes, A. Preto, A. Cavaco-Paulo, Nanomedicin: NBM 2016, 12, 1113.

[15] Y. Liu, Y. Xu, Z. Zhang, Y. Huo, D. Chen, W. Ma, K. Sun, G. Y. Tonga, G. Zhou, D. 
S. Kohane, K. Tao, Nano Lett. 2019, 19, 5515.

[16] M. Shamsipur, A. Barati, S. Karami, Carbon 2017, 124, 429.

[17] S. Baker, G. Baker, Angew. Chem. Int. Ed. 2010, 49, 6726.

[18] X. T. Zheng, A. Ananthanarayanan, K. Q. Luo, P. Chen, Small 2015, 11, 1620.

[19] S. Y. Lim, W. Shen, Z. Gao, Chem. Soc. Rev. 2015, 44, 362.

[20] S. A. Chechetka, E. Miyako, ChemistrySelect 2016, 1, 608.

[21] S. A. Chechetka, E. Miyako, Chem. Lett. 2016, 45, 854.

[22] (a) J. Ge, M. Lan, B. Zhou, W. Liu, L. Guo, H. Wang, Q. Jia, G. Niu, X. Huang, H.

Zhou, X. Meng, P. Wang, C. Lee, W. Zhang, X. Han, Nat. Commun. 2014, 5, 4596.

[23] D. Gao, H. Zhao, X. Chen, H. Fan, Mater. Today Chem. 2018, 9, 103.

[24] K. Hola, Y. Zhang, Y. Wang, E. P. Giannelis, R. Zboril, A. L. Rogach, Nano Today $2014,9,590$

[25] Y.-H. Chien, K. K. Chan, T. Anderson, K. V. Kong, B. K. Ng, K.-T. Yong, Adv. Ther. 2019, 2, 1800090 .

[26] L. Guo, J. Ge, P. Wang, Photochem. Photobiol. 2018, 94, 916.

[27] L. Guo, G. Niu, X. Zheng, J. Ge, W. Liu, Q. Jia, P. Zhang, H. Zhang, P. Wang, Adv. Sci. 2017, 4, 1700085 .

[28] Q. Jia, J. Ge, W. Liu, X. Zheng, S. Chen, Y. Wen, H. Zhang, P. Wang, Adv. Mater. 2018, 30, 1706090.

[29] D. Li, P. Jing, L. Sun, Y. An, X. Shan, X. Lu, D. Zhou, D. Han, D. Shen, Y. Zhai, S. Qu, R. Zbořil, A. L. Rogach, Adv. Mater. 2018, 30, 1705913.

[30] G. Xu, X. Bao, J. Chen, B. Zhang, D. Li, D. Zhou, X. Wang, C. Liu, Y. Wang, S. Qu, 
Adv. Healthc. Mater. 2019, 8, 1800995.

[31] M. Triesscheijn, P. Baas, J. H. M. Schellens, F. A. Stewart, Oncologist 2006, 11, 1034.

D. Pan, J. Zhang, Z. Li, M. Wu, Adv. Mater. 2010, 22, 734.

[33] K. Byrappa, T. Adschiri, Prog. Cryst. Growth Charact. Mater. 2007, 53, 117.

[34] C. Spinato, A. Perez Ruiz de Garibay, M. Kierkowicz, E. Pach, M. Martincic, R.

Klippstein, M. Bourgognon, J. T.-W. Wang, C. Ménard-Moyon, K. T. Al-Jamal, B.

Ballesteros, G. Tobias, A. Bianco, Nanoscale 2016, 8, 12626.

G. Baier, D. Baumann, J. M. Siebert, A. Musyanovych, V. Mailänder, K. Landfester,

Biomacromolecules 2012, 13, 2704.

[36] A. Gabizon, A. T. Horowitz, D. Goren, D. Tzemach, F. Mandelbaum-Shavit, M. M.

Qazen, S. Zalipsky, Bioconjug. Chem. 1999, 10, 289.

[37] S. D. Boss, T. Betzel, C. Müller, C. R. Fischer, S. Haller, J. Reber, V. Groehn, R.

Schibli, S. M. Ametamey, Bioconjug. Chem. 2016, 27, 74.

[38] Y. Li, W. Zhang, L. Zhang, J. Li, Z. Su, G. Wei, Adv. Mater. Interfaces 2017, 4, 1600895.

[49] P. Huang, J. Lin, X. Wang, Z. Wang, C. Zhang, M. He, K. Wang, F. Chen, Z. Li, G.

Shen, D. Cui, X. Chen, Adv. Mater. 2012, 24, 5104.

[40] T. L. Moore, L. Rodriguez-lorenzo, V. Hirsch, S. Balog, D. Urban, C. Jud, B.

Rothen-rutishauser, A. Petri-fink, Chem. Soc. Rev. 2015, 44, 6287.

[41] C. Dubernet, P. Couvreur, Adv. Drug Deliv. Rev. 2002, 54, 631.

[42] J. Shi, P. W. Kantoff, R. Wooster, O. C. Farokhzad, Nat. Rev. Cancer 2016, 17, 20. 
F. Alheid, D. R. Mccrimmon, I. Szleifer, M. C. Hersam, Nano Lett. 2010, 10, 1664.

[44] L. Yildirimer, N. T. K. Thanh, M. Loizidou, A. M. Seifalian, Nano Today 2011, 6, 585.

A. Albanese, W. C. W. Chan, ACS Nano 2011, 5, 5478.

Baidakova, D. A. Kirilenko, S. V. Koniakhin, V. V. Shnitov, V. V. Sokolov, P. N.

Brunkov, A. T. Dideikin, Y. M. Sgibnev, L. Y. Mironov, D. A. Smirnov, A. Y. Vul', V. G.

Golubev, Nanoscale 2018, 10, 13223.

[47] S. Behzadi, V. Serpooshan, W. Tao, M. A. Hamaly, M. Y. Alkawareek, E. C.

Dreaden, D. Brown, A. M. Alkilany, O. C. Farokhzad, M. Mahmoudi, Chem. Soc. Rev. 2017, 46, 4218.

[48] M. Zhu, G. Nie, H. Meng, T. Xia, A. Nel, Y. Zhao, Acc. Chem. Res. 2013, 46, 622.

[49] H. Heinz, C. Pramanik, O. Heinz, Y. Ding, R. K. Mishra, D. Marchon, R. J. Flatt, I.

Estrela-Lopis, J. Llop, S. Moya, R. F. Ziolo, Surf. Sci. Rep. 2017, 72, 1.

[50] M. Lan, S. Zhao, Z. Zhang, L. Yan, L. Guo, G. Niu, J. Zhang, J. Zhao, H. Zhang, P.

Wang, G. Zhu, C.-S. Lee and W. Zhang, Nano Res., 2017, 10, 3113-3123.

[51] L. Guo, G. Niu, X. Zheng, J. Ge, W. Liu, Q. Jia, P. Zhang, H. Zhang and P. Wang, Adv. Sci., 2017, 4, 1700085.

Tian and H. Bi, Small, 2020, 2000680, 1-9. 
[54] J. R. Harbour, S. L. Issler, J. Am. Chem. Soc. 1982, 104, 903.

[55] W. Wang, M. N. Schuchmann, H.-P. Schuchmann, W. Knolle, J. von Sonntag, C. von Sonntag, J. Am. Chem. Soc. 1999, 121, 238.

[56] Z. Li, D. Wang, M. Xu, J. Wang, X. Hu, S. Anwar, A. C. Tedesco, P. C. Morais and H. Bi, J. Mater. Chem. B, 2020, 8, 2598-2606.

[57] L. V. Lutkus, S. S. Rickenbach and T. M. McCormick, J. Photochem. Photobiol. A Chem., 2019, 378, 131-135.

[58] Q. Jia, J. Ge, W. Liu, L. Guo, X. Zheng, S. Chen, M. Chen, S. Liu, L. Zhang, M. Wang, H. Zhang and P. Wang, Adv. Healthc. Mater., 2017, 6, 1601419.

[59] C. Felip-León, M. Puche, J. F. Miravet, F. Galindo, M. Feliz, Mater. Lett. 2019, 251, 45.

[60] (a) Y. Choi, S. Kim, M. Choi, S. Ryoo, J. Park, D. Min, B. Kim, Adv. Funct. Mater. 2014, 24, 5781; (b) Y. Song, W. Shi, W. Chen, X. Li, H. Ma, J. Mater. Chem. 2012, 22, 12568.

[61] T. Kushibiki, Y. Tu, A. O. Abu-Yousif, T. Hasan, Sci. Rep. 2015, 5, 13114.

[62] X. Shi, H. Meng, Y. Sun, L. Qu, Y. Lin, Z. Li, D. Du, Small 2019, 1901507.

[63] J. Ge, Q. Jia, W. Liu, M. Lan, B. Zhou, L. Guo, H. Zhou, H. Zhang, Y. Wang, Y. Gu, X. Meng, P. Wang, Adv. Healthc. Mater. 2016, 5, 665. 


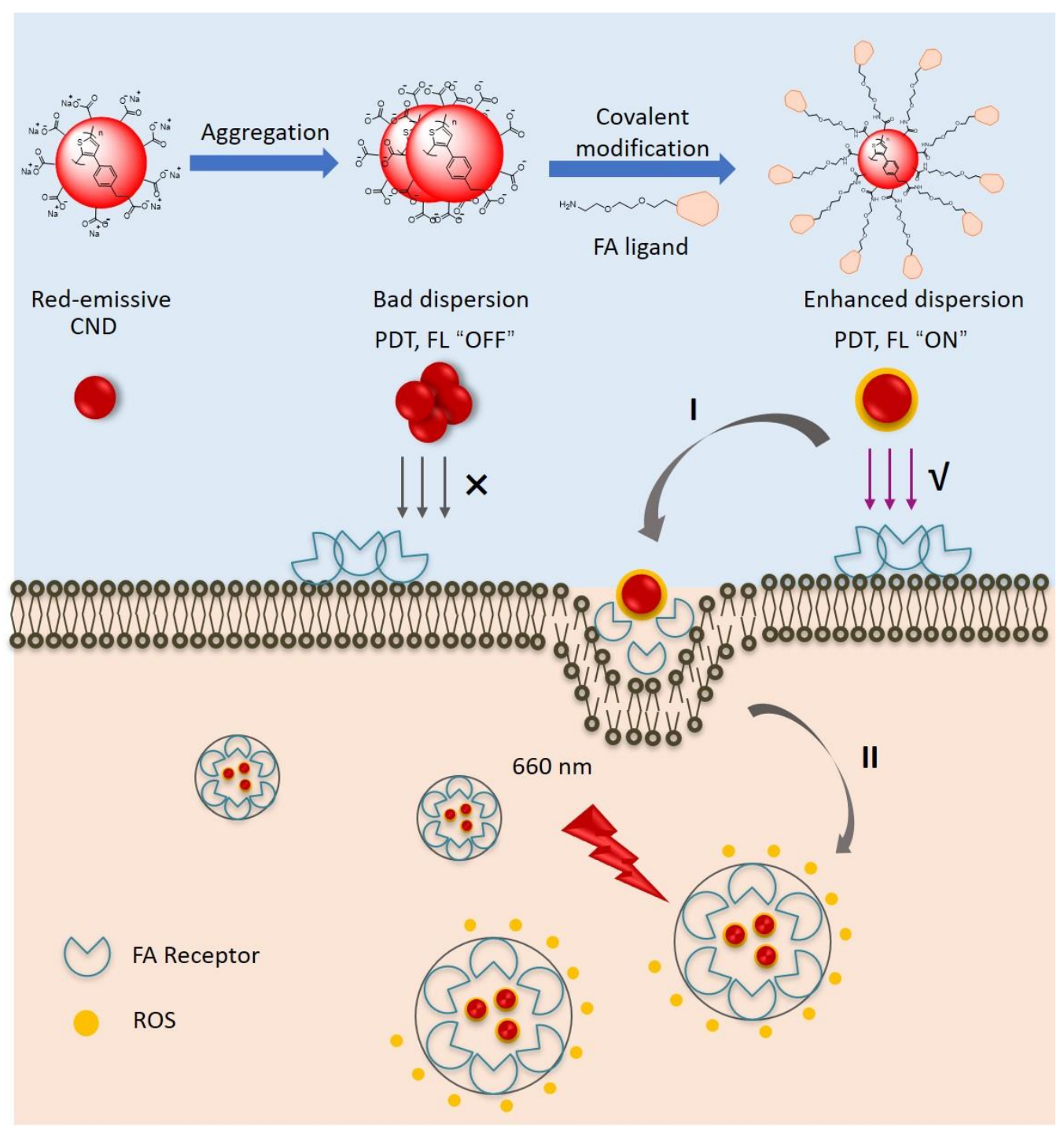

Figure 1. Schematic illustration of the covalent functionalization of RCNDs and their targeted intracellular production of ROS through (I) FR-mediated endocytosis and (II) light irradiation. FL stands for fluorescence. 


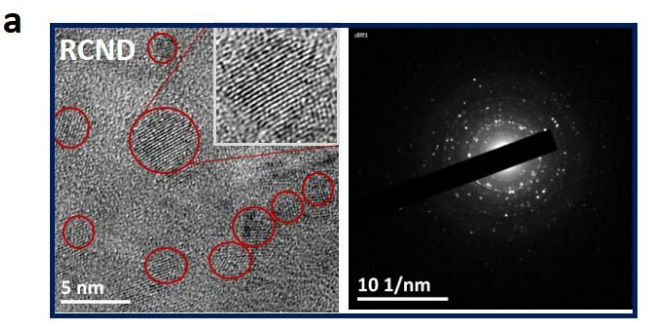

b

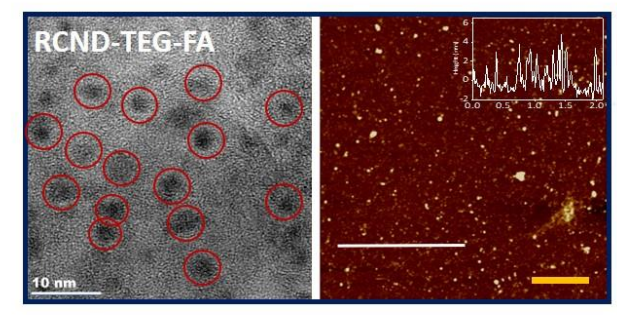

C

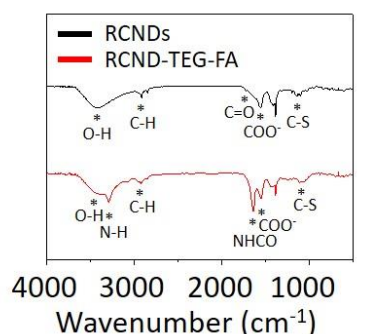

d

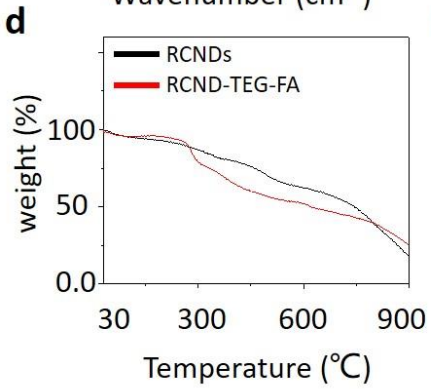

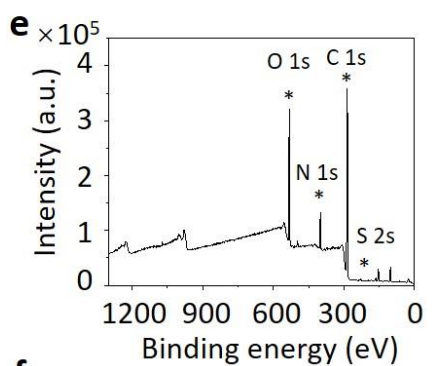

$f$

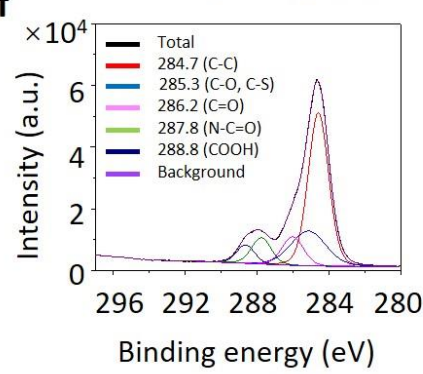

Figure 2. a) HRTEM image (left) and SAED pattern (right) of RCNDs; b) HRTEM (left) and AFM images (right) of RCND-TEG-FA; c) FTIR spectra of RCNDs, FA ligand and RCND-TEG-FA; d) TGA of RCNDs and RCND-TEG-FA; and e) XPS spectra of survey and f) high resolution C1s for RCND-TEG-FA. 

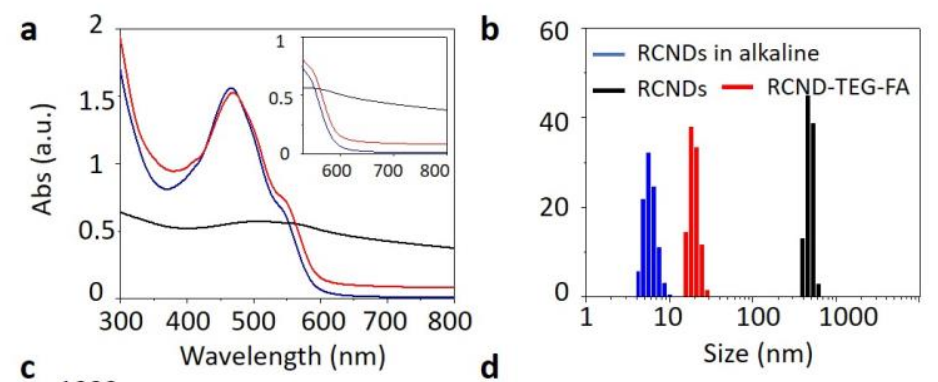

e
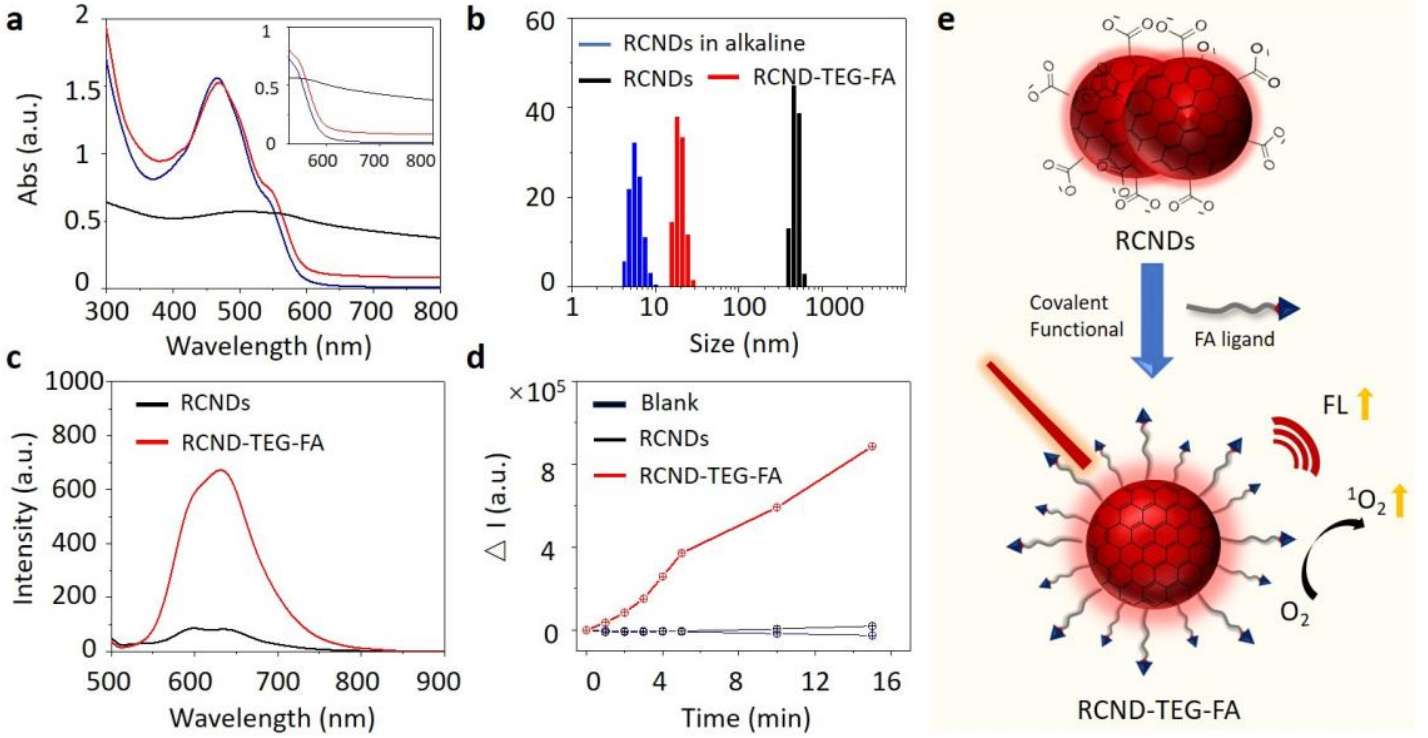

Figure 3. a) Stacked UV-vis spectra and b) DLS of RCNDs in alkaline solution at $\mathrm{pH}=12$ (blue line), RCNDs in water (black line) and RCND-TEG-FA in water (red line); c) The FL spectra of RCND (25 $\left.\mu \mathrm{g} \cdot \mathrm{mL}^{-1}\right)$ and RCND-TEG-FA (25 $\mu \mathrm{g} \cdot \mathrm{mL}^{-1}$ of RCNDs) in water; d) Plotting the fluorescence intensity of DHR123 at $525 \mathrm{~nm}$ in the presence of RCNDs (25 $\left.\mu \mathrm{g} \cdot \mathrm{mL}^{-1}\right)$ and RCND-TEG-FA $\left(25 \mu \mathrm{g} \cdot \mathrm{mL}^{-1}\right.$ of RCNDs) upon $660 \mathrm{~nm}$ laser irradiation $(0.1 \mathrm{~W}$ $\cdot \mathrm{cm}^{-2}$ ) with time; e) Scheme of the covalent functionalization of carbon nanodot aggregates; 

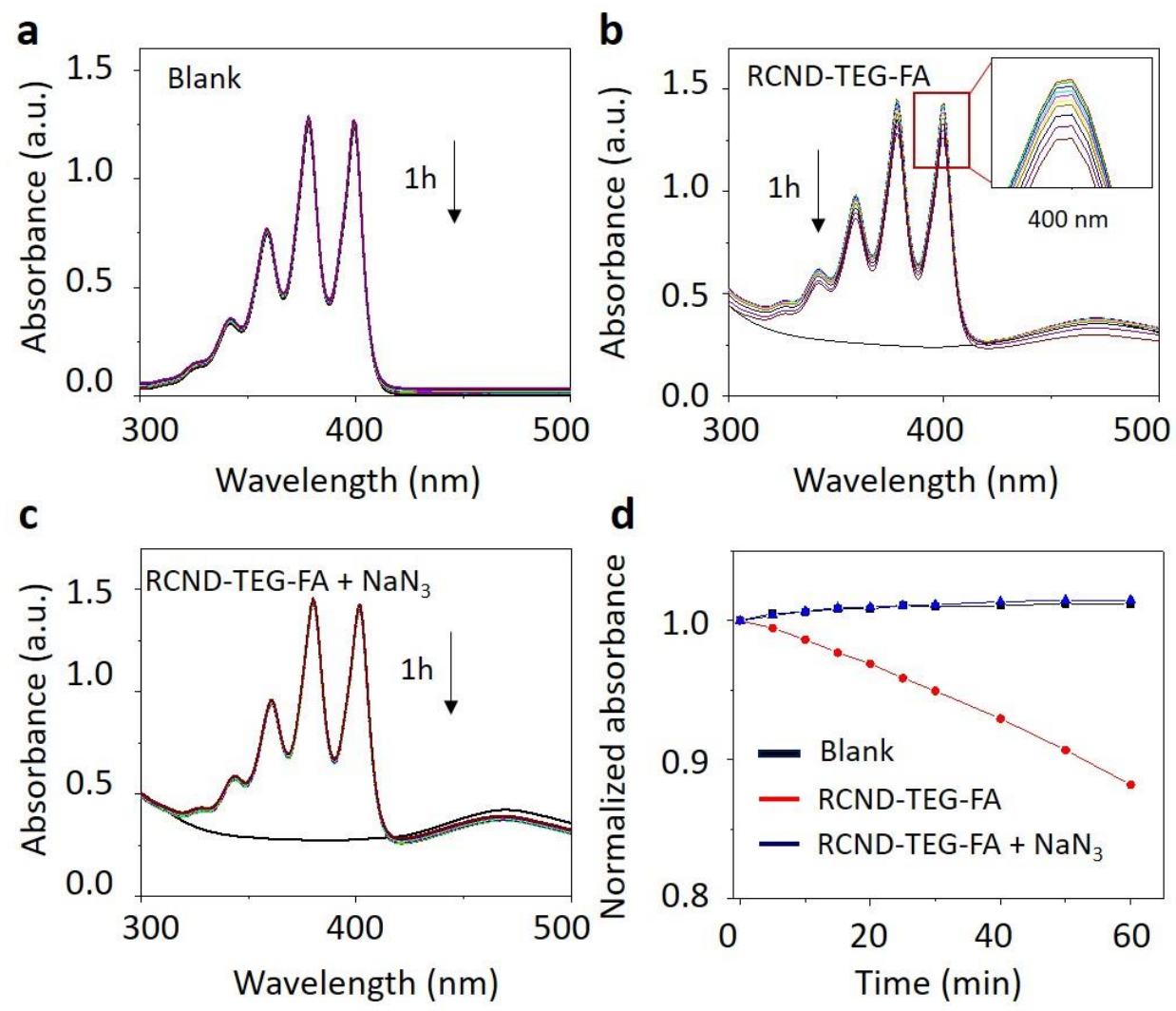

Figure 4. Singlet oxygen characterization. Time-dependent absorbance of the ABDA in different conditions. a) blank; b) RCND-TEG-FA $\left(25 \mu \mathrm{g} \cdot \mathrm{mL}^{-1}\right)$, insert image to show the peak at $400 \mathrm{~nm}$; c) RCND-TEG-FA with adding $5 \mathrm{mg} \mathrm{NaN}_{3}$; d) Normalized absorbance of ABDA at $400 \mathrm{~nm}$ in the presence of RCND-TEG-FA in different conditions. 


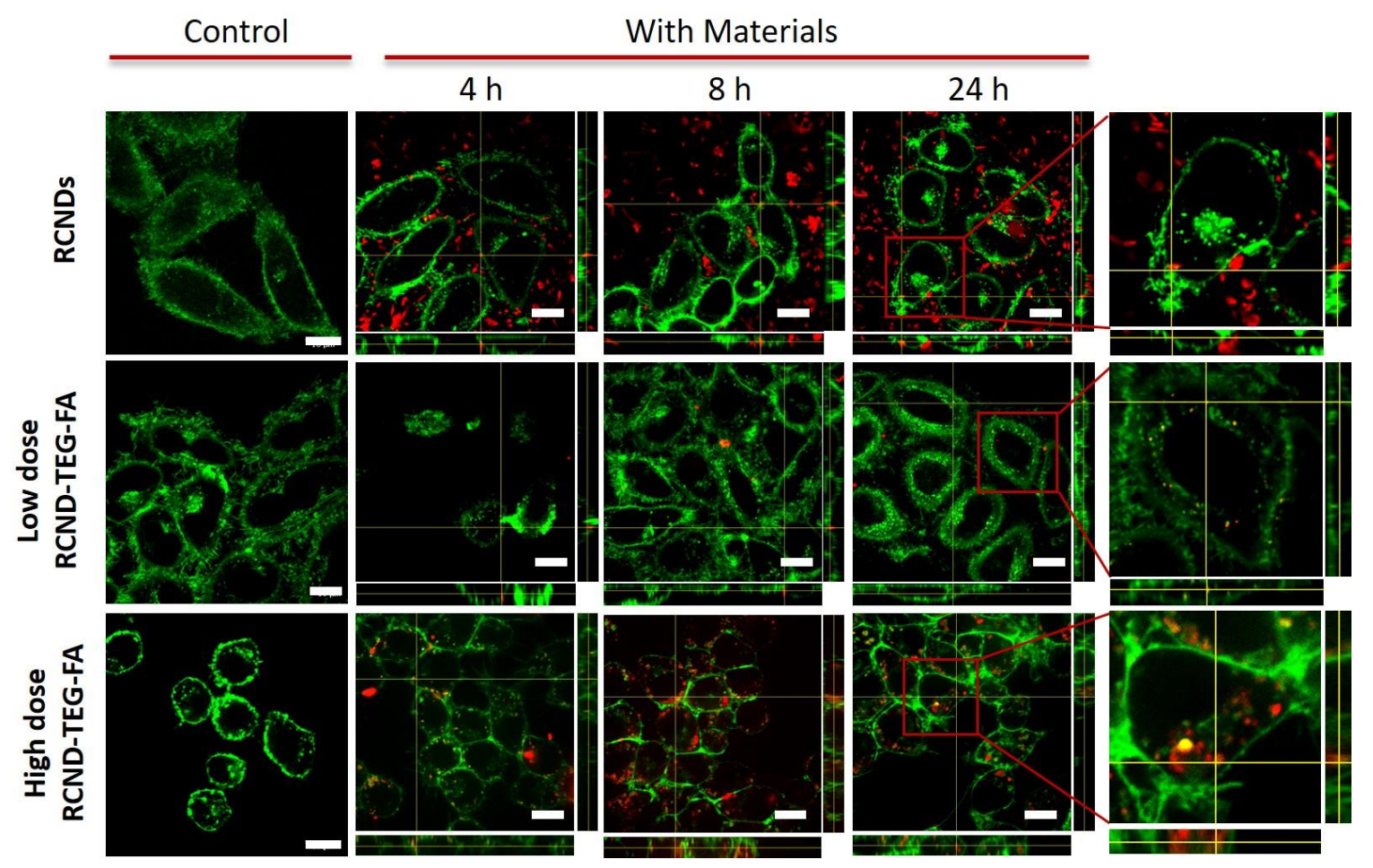

Figure 5. $z-$ Stacking analysis with $x z$ and yz orthogonal views on live HeLa cells incubated with $20 \mu \mathrm{g} \cdot \mathrm{mL}^{-1}$ of RCNDs, and $20 \mu \mathrm{g} \cdot \mathrm{mL}^{-1}$ or $100 \mu \mathrm{g} \cdot \mathrm{mL}^{-1}$ RCND-TEG-FA (upper panel) and UT (lower panel) after 4, 8, and $24 \mathrm{~h}$. In green, membranes stained with CellMask (excitation channel: $488 \mathrm{~nm}$, emission: 500-550 nm); in red, RCND-TEG-FA (excitation channel: $405 \mathrm{~nm}$, emission: $665-715 \mathrm{~nm}$ ). The scale bar is $10 \mu \mathrm{m}$; 

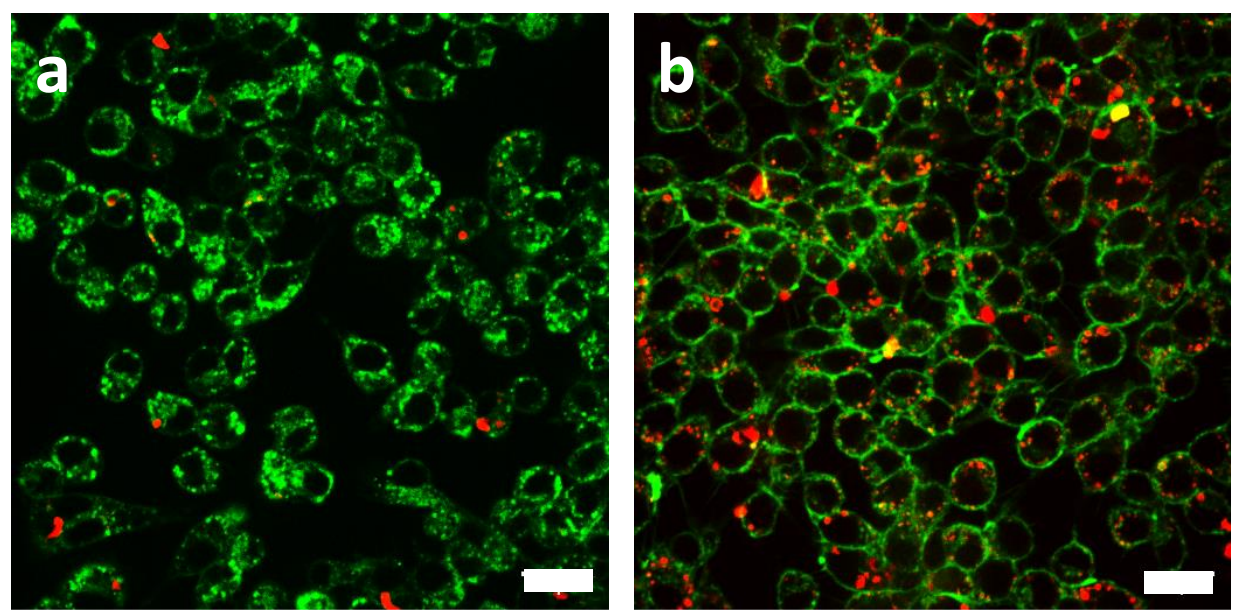

Figure 6. Fluorescence images of $\mathrm{HeLa}$ cells incubated with $100 \mu \mathrm{g} \cdot \mathrm{mL}^{-1}$ of RCND-TEG-FA in the presence (a) or the absence (b) of free FA pretreatment. In green, membranes stained with CellMask (excitation channel: $488 \mathrm{~nm}$, emission: 500-550 nm); in red, RCND-TEG-FA. The scale bar is $20 \mu \mathrm{m}$. 

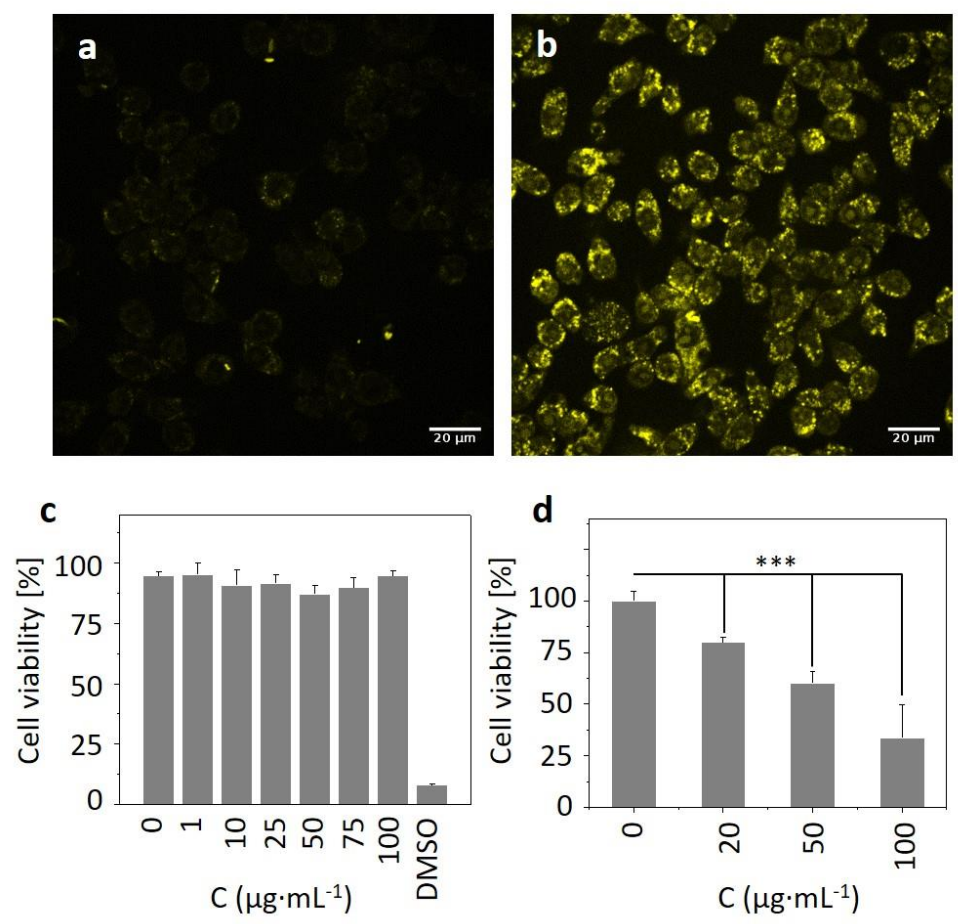

Figure 7. Intracellular ROS formation without (a) or with (b) a low-dose $660 \mathrm{~nm}$ laser irradiation $\left(0.1 \mathrm{~W} \cdot \mathrm{cm}^{-2}\right)$. HeLa cells were incubated with CellROX reagent (excitation channel: $488 \mathrm{~nm}$, emission: $500-550 \mathrm{~nm}$ ) for $30 \mathrm{~min}$ before irradiation. The scale bar is $20 \mu \mathrm{m}$; Cell viability of HeLa cells incubated with increased concentration of RCND-TEG-FA $\left(0-100 \mu \mathrm{g} \cdot \mathrm{mL}^{-1}\right)$ (c) without laser irradiation or (d) under 660 laser irradiation $\left(0.1 \mathrm{~W} \cdot \mathrm{cm}^{-2}\right)$. Data are the mean \pm sd of three independent experiments with each treatment performed in three wells (one-way analysis of variance followed by Tuckney's post-test: ***p $<0.001$ ). 